\title{
A Study to Identify the People with Risk of Obstructive Sleep Apnea and Assess the Prevalence of Hypertension among them in Selected Community Area, Kancheepuram District, Tamilnadu, India
}

\author{
Pillanni Shri Pill Topu ${ }^{1}$, Pushpakala K.J ${ }^{2}$, M.Sc $(\mathbf{N})^{2}$ \\ ${ }^{1} \mathrm{M} . \mathrm{Sc}(\mathrm{N})$ II year- Cardiothoracic Nursing Department, \\ ${ }^{2}$ Associate Professor, Cardio Thoracic Nursing Department, Chettinad Hospital and Research Institute, Tamilnadu, India
}

\begin{abstract}
Hypertension is estimated to cause 7.5 million deaths, about 12.8\% of the total of all deaths. Several epidemiological studies have identified obstructive sleep apnea (OSA) as a risk factor for systemic hypertension. The prevalence is found to be more in developed and in developing countries. India being a fast developing country has prevalence of OSA also rising. Aim: The aim of the study is identify people with risk of OSA and assess the prevalence of hypertension among them. Methods: Quantitative descriptive approach, non experimental descriptive design. Using purposive sampling technique of 101 samples was taken. Identification of people with risk of OSA was done by using STOP bang Questionnaire to participant until it has reach the 101, and the blood pressure was monitored for 3 consecutive week and has been classified according to AHA standard scale. The result shown that $26 \%$ of the samples had high risk of OSA, 64\% of the subjects had intermediate risk of OSA and remaining 10(9.9\%) of the subjects had low risk of OSA among study population. The prevalence of hypertension was $61 \%$ among people with risk of OSA, The study result reveals that the odds of Hypertension were 2.63(3) times higher in people with high or intermediate risk of OSA, when compared to people with low risk of OSA. The correlation coefficient between BMI and stop bang score was $r=0.011$ and $p$ value 0.302 . The correlation coefficient between mean systolic BP and stop bang score was $r=0.075$ and $p$ value 0.006. The correlation coefficient between mean Diastolic BP and stop bang score was $r=0.053$ and $p$ value 0.020 .The result reveals that there is a positive correlation between biophysical parameters with stop bang score. Thus the study suggest OSA may be one of the factor for resistant hypertension, early screening of OSA with proper treatment can be effective in reducing blood pressure.
\end{abstract}

Keyword: OSA, Hypertension, Risk, Prevalence

\section{Introduction}

Health is wealth, health always come first before any other prior. In this fast running generation people giving less important to their health which is taking ultimate turnover with major health issues especially cardiovascular diseases. A nurse, the first line care givers in hospital as well as community who touches not only the patient problem but to their soul too. She's not only a care giver but can be a investigator to bring well being of people. Hypertension is considering one of the major diseases and becoming a fast growing cardiovascular disease in community. Many drug as well alternative therapy has been taken place but there is no reduction in morbidity rate. Globally, the overall prevalence of Hypertension $25 \%$ and in India prevalence was around $40 \%$ in 2008 . The proportion of the world's population with high blood pressure, or uncontrolled hypertension, fell modestly between 1980 and 2008. However, because of population growth and ageing, the number of people with uncontrolled hypertension rose from 600 million in 1980 to nearly 1 billion in 2008

OSA affects $2-4 \%$ of adults, is associated with increased cardiovascular risks and mortality, and increases with age and obesity. The signs and symptoms, and consequences of OSA may be due to physiological changes from repetitive collapse of the upper airway leading to sleep fragmentation, hypoxemia, hypercapnia, changes in intrathoracic pressure, and increased sympathetic activity. Obstructive Sleep Apnoea (OSA) is an independent risk factor for Hypertension and the severity of Hypertension has been shown to increase with the severity of Obstructive Sleep Apnoea. So, the investigator conducted a study to test the hypothesis i.e. whether OSA is a independent confounding factors to elevated blood pressure.

\section{Objective of the Study}

- To identify the people with risk of obstructive sleep apnea in selected community area.

- To assess the prevalence of hypertension among people with risk of obstructive sleep apnea.

- To associate the risk of obstructive sleep apnea with the prevalence of hypertension.

- To correlate the Biophysical parameter with Risk of obstructive sleep apnoea.

- To associate risk of obstructive sleep apnea and the prevalence of hypertension with selected socio demographic variables. 


\section{International Journal of Science and Research (IJSR) \\ ISSN (Online): 2319-7064}

Index Copernicus Value (2013): 6.14 | Impact Factor (2015): 6.391

\section{Methods}

Quantitative descriptive approach, non experimental descriptive design was selected for this study and conducted in Poonjery community rural area located in outskirts of Chennai. A pilot study was conducted prior to the main study with 10 samples and feasibility and practicability of tools and methods was identified. The main study was conducted among calculated sample size 101.Samples were selected by using purposive sampling technique. By using STOP BANG questionnaire the people with risk of Obstructive sleep apnoea was identified and selected as sample who fulfils the sampling criteria. The objective of the study was explained and Informed consent was obtained from the participant. Demographic data was collected by using structured questionnaire and measured biophysical parameters by visiting the samples residence.

Validity of the aneroid sphygmomanometer instrument was done by comparing the blood pressure measurements for four individuals with standard instrument used by the physician and found to be the same during each measurement. The tool was tested for reliability by using Test retest method.

\section{Data Collection and Analysis}

The study was approved by the Institutional Human Ethical Committee prior to the conduction of study. The aim of the study was explained to the study participants and informed consent was obtained by explaining in their own vernacular language. Descriptive statistics method such as frequencies and percentage was used to assess the demographic variables, Risk of OSA and the prevalence of Hypertension. The correlation between the biophysical parameters and risk of OSA was calculated by Karl Pearson coefficient of correlation. The association between the risk of OSA and prevalence of hypertension was calculated by odds ratio and chi square. The association between the risk of OSA and prevalence of hypertension with selected socio demographic variables was evaluated by inferential statistical method- Chi square.

\section{Results and Discussion}

The majority of samples i.e. $67 \%$ of them were in the age of $51-60$ years, $23 \%$ of them were $41-50$ years and $10 \%$ in the age of $31-40$ years. Male were about $58 \%$ and female is about $42 \%$. Majority (72\%) of the study population had completed primary schooling, High school, higher secondary and Graduation and above, was $14 \%, 8 \%$ and $6 \%$ respectively, nuclear family was $59(58 \%)$ and the remaining $42(42 \%)$ belonged to joint family. The occupation was sedentary in nature in $8 \%, 52 \%$ people were working in occupations with moderate physical activity and the remaining $41 \%$ were working in occupations with heavy physical activity. Number of people reporting regular exercise was only $9 \%$ and the remaining majority $91 \%$ of them was not doing any exercise. 34\% have reported walking daily and remaining $66 \%$ of them don't have habit of daily walk.
Among the study population, $26 \%$ of people had high risk of OSA, $64 \%$ of the people had intermediate risk of OSA and remaining $10 \%$ of the subjects had low risk of OSA. A similar study conducted in semi-urban Delhi population suggested OSA prevalence to be $19.7 \%$ in men and $7.4 \%$ in women. Another study conducted in northern India revealed an overall prevalence of $9.3 \%$ for OSA with $13.5 \%$ in male and $5.5 \%$ in female. These studies suggest that OSA is undoubtedly increasing in India. The study concludes that Lifestyle modifications have resulted in an increase in prevalence of OSA among Indian population.

Among the people with of OSA, $61 \%$ of them had hypertension and $39 \%$ of them don't have hypertension. According to AHA standard, the prevalence of hypertension was $61 \%$ in study population. The prevalence of pre hypertension was $24 \%$ and the prevalence of stage -1 , stage 2 and hypertensive crisis was $67 \%, 3 \%$ and $6 \%$ respectively among people with OSA. Association between Prevalence of Hypertension with Risk of Obstructive Sleep Apnea result reveals that the odds of Hypertension were 2.63 times higher in people with high or intermediate risk of OSA, when compared to people with low risk of OSA. The $95 \%$ confidence interval of Odds ratio was 0.69 to 10.02 and the $\mathrm{P}$ - value was 0.14 .

A similar cohort study conducted to identify the association between Blood pressure and sleep disorder in 2000 and 2001 and in 2005 and 2006. Sleep duration and sleep maintenance (a component of sleep quality) were calculated. Analysis included 578 African Americans and whites aged 33 to 45 years at baseline. Outcome measures were systolic BP (SBP) and diastolic BP (DBP) levels, 5-year change in BP, and incident hypertension. The study concluded reduced sleep duration and consolidation predicted higher BP levels and adverse changes in BP, suggesting the need for studies to investigate whether interventions to optimize sleep may reduce $\mathrm{BP}$.

\section{Correlation between Selected Biophysical Parameter with Risk of OSA}

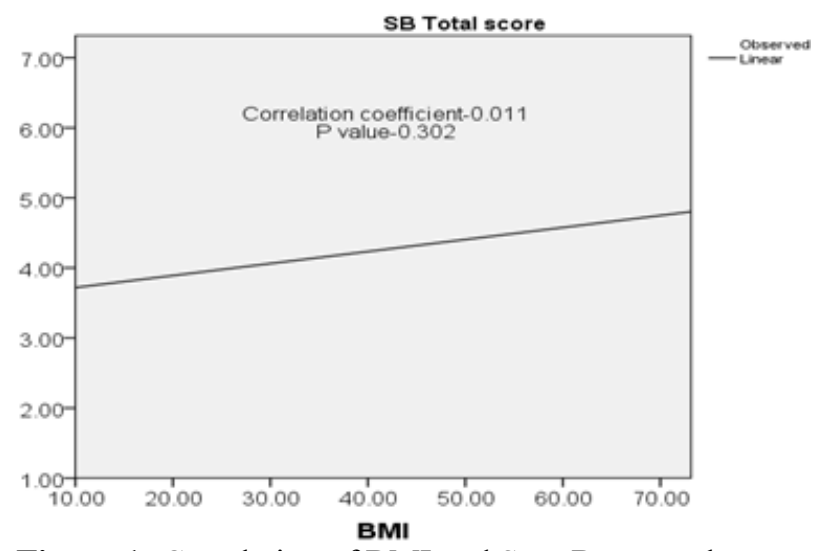

Figure 1: Correlation of BMI and Stop Bang total score 


\section{International Journal of Science and Research (IJSR) \\ ISSN (Online): 2319-7064}

Index Copernicus Value (2013): 6.14 | Impact Factor (2015): 6.391

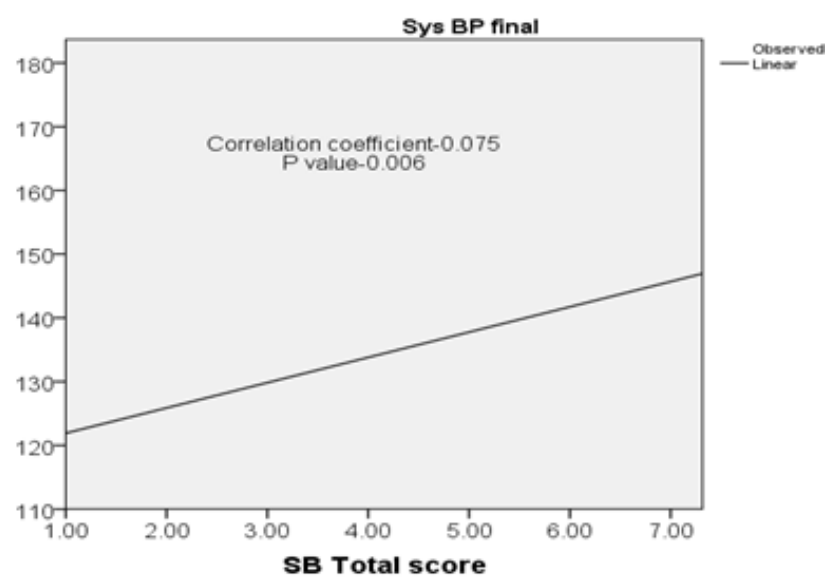

Figure 2: Correlation of Stop bang total score and Mean Systolic BP

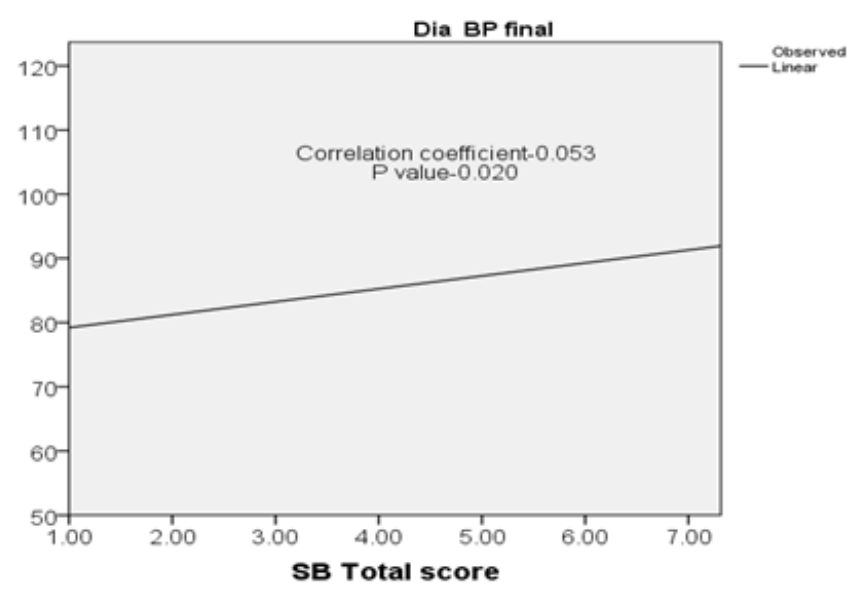

Figure 3: Correlation of Stop bang total score and Mean Diastolic BP

The result reveals that there is a positive correlation between biophysical parameters with Risk of Obstructive sleep apnea.

\section{Implications for Nursing Practice and Research}

The present study will enable, nurses to apply theory into practice as create awareness about obstructive sleep apnea and early identification of hypertension among them in the community. Nurses can conduct more research studies on prevalence of obstructive sleep apnea and identify how it act as a indepependent confounding risk factor for other diseases, and also publish articles in the journal explaining the importance of early detection and prevention of hypertension due to obstructive sleep apnea. There is a need for extensive and intensive research in this area to provide evidence based care to bring out the best in care of patient.

\section{Conclusion}

Based on the objectives there was a significant association between risk of OSA with hypertension. The study result concludes that, there is more number of people with risk of OSA in general population and the study also shows that the prevalence of hypertension is high among the people with risk of obstructive sleep apnea. So it's essential to insist health professionals to create awareness among public about risk of Obstructive Sleep Apnea and consider OSA as one of the risk factor for hypertension.

\section{References}

[1] M. A. Martinez-Garcia, R. Gomez-Aldaravi, J. J. SolerCataluna,T. G. Martinez, B. Bernacer-Alpera, and P. Roman-Sanchez. Positive effect of CPAP treatment on the control of difficult-to treat hypertension. European Respiratory Journal.2007. 29(5)

[2] 951-957.

[3] L. J. Epstein, D. Kristo, P. J. Strollo et al.Clinical guideline for the evaluation, management and long-term care of obstructive sleep apnea in adults. Journal of Clinical Sleep Medicine.2009. 5(3). 263-276.

[4] T. Young, P. E. Peppard, and D. J. Gottlieb.Epidemiologyof obstructive sleep apnea: a population health perspective. American Journal of Respiratory and Critical CareMedicine.2002 .165(9).1217-1239.

[5] .P. E. Peppard, T. Young, M. Palta, and J. Skatrud.Prospective study of the association between sleep-disordered breathing and hypertension. New England Journal of Medicine.2000. 342(19) .13781384.

[6] P. Lavie, P. Herer, and V. Hoffstein.Obstructive sleep apnea syndrome as a risk factor for hypertension: population study. British Medical Journal.2000.320 (7233). 479-482.

[7] J. M. Marin, A. Agusti, I. Villar et al.Association between treated and untreated obstructive sleep apnea and risk of hypertension. Journal of the American Medical Association.2012.307 (20). 2169-2176.

[8] R.Wolk, A. S. M. Shamsuzzaman, and V. K. Somers.Obesity, sleep apnea, and hypertension.Hypertension.2003. 42(6).1067-1074.

[9] G. Logan, S. M. Perlikowski, A.Mente et al.High prevalence of unrecognized sleep apnea in drugresistant hypertension.Journal of Hypertension.2001.19 (12).2271-2277.

[10].S. C. Gonc,alves, D. Martinez. Gus et al.Obstructive sleep apnea and resistant hypertension: a case-control study. Chest.2007. 132(6) .1858-1862.

[11] M.Gus, S. C.Gonc,alves, D.Martinez et al.Risk for obstructive sleep apnea by Berlin Questionnaire, but not daytime sleepiness, is associated with resistant hypertension: a case-control study. American Journal of Hypertension. 2008. 21(7). 832-835.

[12] Srihari R, Mathangi D. C. Shyamala R. OSA: an awakening disorder. IOSR journal.2014. 4 (4).4-7.

[13] Kristen L. Knutson, Eve Van Cauter, Paul J. Rathouz, Lijing L. Yan, Stephen B. Hulley, Kiang Liu, Diane AL. Association Between Sleep and Blood Pressure in Midlife .The CARDIA Sleep Study .2009.169(11):1055-1061. 